\title{
Pseudoaneurysm of the Femoral Artery - An Unusual Presentation of Behçet's Disease
}

\author{
Susy Kovatz*, Ramona Segal, Lotan Shilo, Alla Shabun and Louis Shenkman \\ Department of Medicine C, Meir Hospital Center, Kfar Saba, Israel and The Sackler Faculty of Medicine, Tel Aviv Uni- \\ versity, Tel Aviv, Israel
}

\begin{abstract}
Behçet's disease is a multisystem disorder of probable autoinflammatory etiology with manifestations that can affect many organ systems. We present a patient with the unusual presentation of splenic infarction and pseudoaneurysm of the iliac artery. Behçet's disease should be included in the differential diagnosis of pseudoaneurysms if they are accompanied by other classic features of this interesting disease.
\end{abstract}

\section{INTRODUCTION}

Behçet's disease is a multisystem disorder of probable autoinflammatory etiology [1] with manifestations that can affect many organ systems. Its classical presentation includes recurrent oral and genital ulcers and skin inflammatory reactivity (pathergy test). The skin also may be involved with folliculitis or erythema nodosum. Eye involvement includes iritis, uveitis retinal vessel occlusions and even optic neuritis [2]. In approximately one-fourth of patients, superficial or deep thrombophlebitis is observed. Neurologic involvement is rare, as is the development of peripheral arterial aneurysms [3]. In this article we describe a patient with an unusual presentation of Behçet disease whose main manifestations were vascular in nature.

\section{DESCRIPTION OF THE CASE}

A 35-year-old man of Moroccan descent entered the hospital with complaints of left inguinal and left testicular pain of one month's duration. During this time he also noted a 7 $\mathrm{kg}$ weight loss, weakness and occasional night sweats. Three days prior to admission he had fever. Until this admission he had been in excellent health. He was a police officer, and related no recent trauma or unusual exertion. His temperature on admission was $38.1^{\circ} \mathrm{C}$, BP $122 / 80$, pulse 92 and regular. Positive physical findings included several small aphthae on the soft palate, shotty tender lymphadenopathy in the left posterior cervical triangle and tenderness in the left inguinal region and left testis. Complete blood count, differential, electrolytes and liver function studies were normal. Erythrocyte sedimentation rate was $80 \mathrm{~mm}$ and CRP was 0.22 $\mathrm{mg} / \mathrm{dl}$.

Because of the history of weight loss, fever and night sweats, computerized tomography of the chest and abdomen was performed, revealing an infarct of the spleen and a pseudoaneurysm of the left iliac artery immediately past its bifurcation.

*Address correspondence to this author at the Department of Medicine C, Meir Hospital Center, Kfar Saba, Israel and The Sackler Faculty of Medicine, Tel Aviv University, Tel Aviv, Israel; E-mail: susyk@clalit.org.il
After blood cultures were obtained, antibiotic therapy was initiated with ampicillin and garamycin for treatment of suspected bacterial endocarditis, and when initial blood cultures returned sterile he was given ceftriaxone as treatment for HACEK organisms. Transthoracic and transesophageal echocardiographs revealed normal cardiac anatomy with no evidence of vegetations. In spite of antibiotic therapy, fevers between $38-39^{\circ}$ persisted for one week. After PCR for bacterial antigens returned negative, antibiotic therapy was discontinued. The patient continued to complain of left inguinal and left back pain, and repeat CT angiography revealed that the pseudoaneurysm had become enlarged. The possibility of vasculitis was raised and serological studies were performed, including p-ANCA, C-ANCA, anti-double-stranded DNA, hepatitis, B, C, HIV, antibodies to antiphospholipids, and HLA B51, all of which were negative.

On the seventh day of admission, the patient had a transient episode of confusion, and CT of the brain and lumbar puncture were performed, both of which were negative.

At this time, the patient was re-questioned, and he related having had oral and genital aphthae several times in the past two years. Ophthalmologic exam showed no evidence of retinal vasculitis or scleritis. Repeated examination revealed the pathergy reaction at several venipuncture sites (small erythematous papules in the antecubital fossae). These findings led to the consideration of Behçet's disease as the cause of the fever, pseudoaneurysm, splenic infarct and aphthae, and treatment was initiated with dexamethasone, $14 \mathrm{mg}$ daily with a rapid clinical response. The vascular consultant recommended surgical repair of the pseudoaneurysm, and a prosthetic graft was inserted successfully to reinforce the affected iliac artery. The patient made an uneventful recovery and was discharged to outpatient care while receiving prednisone, $20 \mathrm{mg}$ daily and cyclophosphamide, $1 \mathrm{mg} / \mathrm{kg}$.

\section{DISCUSSION}

Although Hippocrates may have been the first to describe the symptoms of the disease [4], the first contemporary de- 
scription of ocular Behçet disease was made by adamantiades, and then independently by Behçet himself [5]. In this article we describe an unusual presentation of Behcet's disease with a large vessel involvement, a pseudoaneurysm of the common iliac artery (CIA). Usually, vascular involvement in Behcet's disease consists of small vessel disease of both arteries and veins. Large vessel involvement occurs in approximately $30 \%$ of patients with vascular disease, and can lead to stenosis, hemorrhage, aneurysm and thrombus formation. Superficial thrombophlebitis and deep vein thrombosis are characteristic findings. Occlusive arterial disease is more common in the lower limbs and may have a better prognosis than that of aneurysm formation [6-8].

Active arteritis occurs initially, followed by destruction of the media and fibrosis. Vasculitis of the vasa vasorum seems to be the major site of pathology. The cessation of nutrient flow to the arterial wall due to inflammatory obliteration of the vasa vasorum can lead to perforation or pseudoaneurysm formation [9].

The characteristic site of large artery involvement is the pulmonary artery. Vascular lesions in the lung may produce recurrent episodes of dyspnea, cough, chest pain and hemoptysis [10].

In a recent description of 33 patients with vascular involvement, 12 had only venous lesions, 11 only arterial lesions and 10 had both. The main angiographic findings were acute or chronic thrombosis in deep veins of the lower extremities (19 patients), inferior vena cava (4 patients) and superior vena cava ( 2 patients). Pseudoaneurysms of large or medium sized arteries were noted in 11 patients, and occlusions/stenoses of distal arteries in 13 patients [8].

Our patient, in addition to the pseudoaneurysm of the CIA, also suffered from recurrent oral aphthae (more then 46 episodes per year), an isolated episode of genital ulcers and a transient confusional state. Pathergy test was positive. After we excluded other etiologies that cause splenic infarction and pseudoaneurysm (such as: salmonella infection, collagen disease, embolic disorders, hematological disorders and trauma) we concluded that we were confronted with an unusual presentation of Behcet's disease, despite the fact that HLA-B51 was negative.

Medical treatment of Behçet disease is based on use of a variety of anti-inflammatory drugs, including topical glucocorticoids for aphthae, aspirin and colchicine, and systemic glucocorticoids for uveitis and CNS involvement. Reducing the activity of IL- $1 \beta$ activity by administration of anti-tumor necrosis factor therapy including anakinra may be useful in recalcitrant cases [11].

The treatment of arterial involvement in Behcet's disease is a combined medical (immunosuppressive drugs with high dose glucocorticoids and cyclophosphamide) and surgical approach or by interventional radiology treatment. The literature in this domain consist of very small case reports and series cases with an inclination for surgical therapy as treatment of choice with resection of the lesion and placement of a polytetrafluoroethylene interposition graft. Surgery must be performed as early as possible, because rupture of an in- flammatory aneurysm can occur even when it is small. Because of the inflammation of the surrounding tissues, surgical repair is often difficult. Arterial anastomoses should be performed only in disease-free segments, which means that intervention should be avoided in the active stage of the disease, if possible [12]. Recurrence following a surgical treatment is about $50 \%$ with aneurysm formation at the anastomotic site and graft occlusion (thrombosis) $[13,14]$. However, suture line reinforcement with a strip of Teflon felt, surgery during the inactive stage of the disease, and the use of autografts can decrease the incidence of pseudoaneurysm formation.

In the last years there has been an increased in the number of percutaneous angioplasties with a technical success rate of stent graft insertion higher then $90 \%$; successful insertion results in the exclusion of the aneurysm and complete thrombosis [15].

But there has to be an awareness of the pathergy phenomenon at the insertion site, and that is why it is suggested that is angiography should be postponed until the inflammatory reaction subsides.

Our patient had undergone a successful angioplasty with stent insertion to the CIA and total exclusion of the pseudoaneurysm by thrombosis.

\section{CONCLUSIONS}

In this article we have presented an unusual and challenging manifestation of Behcet's disease, and raised the awareness that the diagnosis of Behçet's disease should always be considered in young adult patients from the Mediterranean region or Asia (the silk road disease) who present with an pseudoaneurysm at an unusual location. Patients with Behçet's disease should be evaluated regularly for early diagnosis of arterial complications, and surgery or endovascular intervention should be considered promptly in any case of risk of rupture.

\section{REFERENCES}

[1] Gül A. Behçet's disease as an autoinflammatory disorder. Curr Drug Targets Inflamm Allergy 2005; 4: 81-3.

[2] Sakane T, Takeno M, Suzuki N, Inaba G. Behcet's disease. N Engl J Med 1999; 341: 1284-91.

[3] International Study Group for Behcet's Disease. Criteria for diagnosis of Behçet's disease. Lancet 1990; 335: 1078.

[4] Tirilomis T. Some more historical notes on Adamantiades- Behçet's disease. Chest 2001; 120: 2116

[5] Cheng TO, Tirilomis T. Behçet disease, adamantiades-Behçet disease, or hippocrates-adamantiades-Behçet disease? Chest 2002; 122: 381-2

[6] Bensaid Y, Ameur A, Kabiri $\mathrm{H}$, et al. Arterial complications of Behcet's disease. Report of 13 cases. J Mal Vasc 1997; 22: 24-8.

[7] Hamza M. Large artery involvement in Behcet's disease. J Rheumatol 1987; 14: 554-9.

[8] Ko GY, Byun JY, Hoi BG, Cho SH. The vascular manifestations of Behçet's disease: angiographic and CT findings. Brit J Radiol 2000; 73: $1270-4$.

[9] Matsumoto T, Uekusa T, Fukuda Y. Vasculo- Behcet's disease: a pathologic study of eight cases. Hum Pathol 1991; 22: 45-51.

[10] Saba D, Saricaoglu H, Bayram AS, et al. Arterial lesions in Behcet's disease. Vasa 2003; 32: 75-81.

[11] Botsios C, Sfriso P, Furlan A, Punzi L. Resistant Behçet's disease responsive to anakinra. Ann Intern Med 2008; 149: 284-6. 
[12] Kuzu MA, Ozaslan C, Koksoy C, Gurler A, Tuzuner A. Vascular involvement in Behcet's disease: 8 year audit. World J Surg 1994; 18: 948-53.

[13] Ozeren M, Mavioglu I, Dogan OV, Yucel E. Reoperation results of arterial involvement in Behcet's disease. Eur J Vasc Endovasc Surg 2000; $20: 512-9$
[14] Tuzun H, Besirli K, Sayin A, et al. Management of aneurysms in Behçet's syndrome: an analysis of 24 patients. Surgery 1997; 121: 150-6.

[15] Park JH, Chung JW, Joh JH, et al. Aortic and arterial aneurysms in Behcet disease: management with stent-grafts-initial experience. Radiology 2001; 220: 745-50.

(C) Kovatz et al.; Licensee Bentham Open.

This is an open access article licensed under the terms of the Creative Commons Attribution Non-Commercial License (http: //creativecommons.org/licenses/ by-nc/3.0/) which permits unrestricted, non-commercial use, distribution and reproduction in any medium, provided the work is properly cited. 